\title{
A digital method for correcting planned electric energy consumption as a step to the Energynet market
}

\author{
Oleg Russkov ${ }^{1 *}$, and Sergei Saradszhivili ${ }^{1}$ \\ ${ }^{1}$ Peter the Great Saint Petersburg Polytechnic University, 195251 Polytechnicheskaya st. 29, Russian \\ Federation
}

\begin{abstract}
Creating the modern high-tech industry should be viewed as a fusion of material and digital production and control methods. Industrial enterprises with uneven electric energy consumption, which have failed to adapt to the requirements of modern digital wholesale electricity market, have to spend significant money to pay for hourly deviations of electric energy, whose price constantly grows. A digital method for correcting planned electric energy consumption based on the theory of games is offered to reduce the costs of these enterprises, which have no possibility for regulating own load on a real time basis. The updating is carried out depending on hourly relations of prices on the wholesale electric energy market in order to exclude the risks of making unprofitable hourly transactions. An algorithm of the proposed method is provided and a predictive model developed on its basis is described and positive results of its testing on the basis of a metallurgical enterprise.
\end{abstract}

\section{Introduction}

Nowadays, digital technologies have found their way into all the areas of human activity. In particular, the digital economy is impossible without high-tech industry based on digital "future factories, within which a product passes through design stage to production at the shortest time and with minimum expenses. Several plans for developing perspective future markets, in particular, cross-industrial "Technet", "Autonet", "Energynet" markets have been approved in Russia to support this strategy at the Government level. Active participation of an electric energy consumer during on-line dispatching control of local energy system using digital algorithms and new generation devices is assumed within the framework of the latter. This will make it possible to develop transparent and accurate planning of loads for electric power generators within all the energy system segments. Within the framework of "Energynet" it is proposed to create digital algorithms and devices allowing optimal self-dispatching of the energy system. Thus, a future consumer will switch over from passive electric energy consumption to on-line participation in minimization of own hourly deviations of planned electric energy consumption from actual one. It should be noted that the task of adequate planning of consumption of any type of

* Corresponding author: zenit-che@mail.ru 
resources has been relevant at all times. The main characteristic determining the accuracy of this planning is an uneven load curve. In particular, a metallurgical enterprise has an uneven nature of electric energy consumption due to the steel melting process peculiarities. Applying the quick-acting computing means and up-to-date predictive algorithms will allow combining the effects of digital methods on the functional process of an industrial enterprise and its economics improving the most important production and economic indicators of the country's entire industrial sectors, thus taking care of environmental problems, because active participation of industrial enterprises in the process of electric energy consumption will make it possible to refuse from loading inefficient electric energy generator by saving non-renewable resources to be used for electric energy generation.

\section{Brief review of existing prediction methods}

There is a great number of methods, which are actively applied to predict electric energy consumption. At present time, mathematical time series models prove to be the best, they include autoregressive models, exponential smoothing models, neural network models, Markov's chain models and those based on classification and regression trees CART [1]. These models are used by industrial enterprises as well in conditions of wholesale electric energy market [2]. However, a significant decrease in predictive accuracy with irregularity of time series are referred to well-known disadvantages of these models [3]. The human factor and non-stationary nature of steel melting process are the main causes of irregular electric energy consumption during metallurgical production [4]. Some metallurgical enterprises, in particular, Oskolsky electrometallurgical plant, try to use neural network models to plan their hourly electric energy consumption [5]. Works on an analysis of hourly electric energy consumed by Magnitogorsk metallurgical plant show the inefficiency of time series models and correlative-regression analysis [6]. For example, Volzhsky pipe plant carries out involuntarily planning activities according to average previous day's consumption based on an expert judgment made by personnel of the chief power engineer department [7]. Foreign researchers ironically call this approach a "straw man's prediction" [8]. Due to no generally accepted solution available for planning irregular consumption there are prediction methods for market price indicators actively developed, which are used to predict intermediate electric energy consumption [9]. Therefore, at present time there is a big field for experiments and improvement of digital methods and models including those within the "Energynet" area.

\section{Cost elements of electric energy consumed by a large enterprise}

Electric energy cost $\mathrm{S}$ on the wholesale energy market (EM) at every hour of day is calculated in accordance with market regulations $[10,11]$ as per the formula below:

$$
S=C_{D A M} \cdot V_{p}+\left\{\begin{array}{l}
\left(V_{f}-V_{p}\right) \cdot C_{B M+}, \text { if }\left(V_{f}-V_{p}\right) \\
\left(V_{f}-V_{p}\right) \cdot C_{B M-}, \text { if }\left(V_{f}-V_{p}\right)
\end{array},\right.
$$

Where $C_{D A M}$ is a price on the day ahead market (DAM), $V_{p}$ is planned, $V_{f}$ is actual consumption, $C_{B M+}$ is a buying price, $C_{B M-}$ is a selling price on the balancing market (BM). 
In this case the EM with the purpose of stimulating the market participants for accurate planning of hourly electric energy consumptions sets the DAM and BM prices in accordance with the following formula:

$$
C_{B M-} \leq C_{D A M} \leq C_{B M+}
$$

Every hour the price of one of the BM prices matches the DAM price (Fig. 1), determining the profitable type of transaction; type $0-C_{D A M}$ coincides with $C_{B M+}$ (a white rectangular), type $1-C_{D A M}$ coincides with $C_{B M-}$ ( a black rectangular):

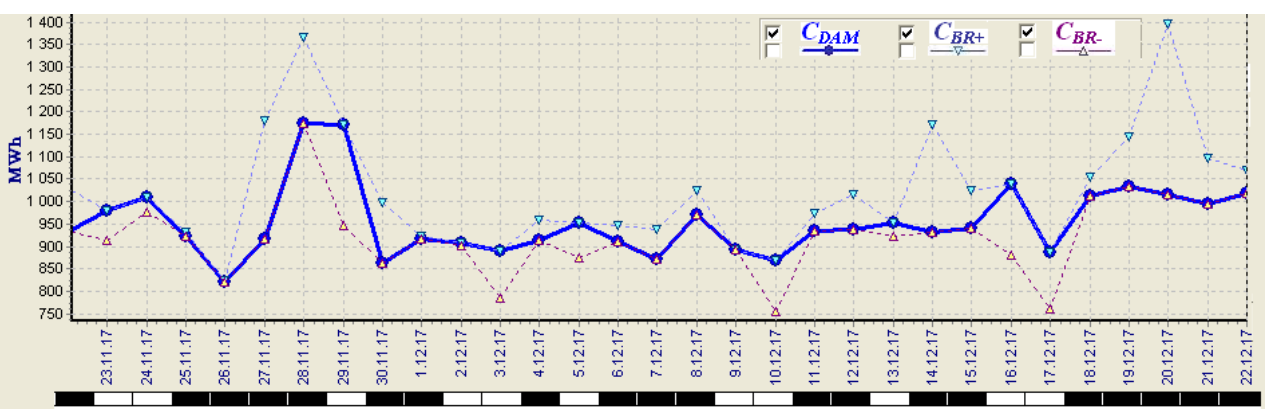

Fig. 1. DAM and BM price ratio for a metallurgical enterprise in Volgograd region at time of day 5:00-6:00.

For an EM consumer there are 2 types of costs related to payment for hourly electric energy deviations.

-total cost of hourly deviation to be paid at high BM prices relative to the DAM ones. This type of costs can be reduced without changing the total volume of deviations.

-cost of EM out-of-balance adjustment interest invoiced by the commercial market operator upon completion of settling period according to a ratio of certain consumer's total hourly deviation volume to all the EM participants' total deviation volume [11]. This type of costs can be reduced by only changing the total volume of consumer electric energy deviations.

The characteristics of enterprise's load curve determine an option to be selected to reduce costs. If the consumer has a relatively even load curve then he can use well recommended existing time series models and reduce the total volume of his deviations. If the consumer can control the load on a real time basis then he will be able to minimize his deviations without any predictive models. If the consumer has an uneven load curve that does not allow planning in-house electric energy consumption by means of existing predictive models [7] as well as if he cannot regulate the load on a real time basis due to reasons that are not related to the production technology then he has to deal with a great amount of electric energy deviations. A metallurgical enterprise has this type of electric energy consumption in particular as it has been mentioned above. Consequently for these EM participants there is only one way to reduce the costs of both types - avoiding unprofitable hourly transactions on the BM by switching over from quantitative prediction of electric energy consumption to qualitative prediction of BM and DAM price ratios. Thus, planned consumption $V_{p}$ is predicted in a vicarious manner. 


\section{Digital method for updating of planned electric energy consumption}

At the first stage the enterprise calculates its load curve for the next days based on the production plans of its main process facilities. The main task of this first stage is to form such a planned hourly load curve that will be close to an average value of actual electric energy consumption on the next day as much as possible. Calculation is carried out according to the following formula:

$$
V_{p}=\frac{\sum_{j=1}^{k} P l_{j}^{*} U_{j}}{24},
$$

Where $\mathrm{j}$ is the serial number of facility, $\mathrm{k}$ is the number of facilities, $P l_{j}$ is the production plan, (tn), $U_{j}$ is the specific consumption of facility $\mathrm{j},(\mathrm{kW} \cdot \mathrm{h} / \mathrm{tn})$.

The preliminarily calculated planned curve for each hour separately is updated at the second stage. In this case it is taken into account that all the EM participants have no full information about DAM requests from each other neither about existing limitations of the unified energy system cross-flows nor about actual changes in demand for electric energy by consumers of their region [10]. Consequently, the enterprise with uneven uncontrolled consumption needs to solve the task of making decisions and selecting the optimal option from a variety of alternate values for updating the planned electric energy consumption in uncertain conditions related to the future ratios of both hourly DAM and BM prices and the volumes of planned and actual electric energy consumption [7]. The mathematical mechanism that is mostly suitable for resolving this type of task is a mechanism of game theory. The EM participant competes to get the maximum profit by sending their requests in attempts to predict the requests sent by other participants (Fig. 2).

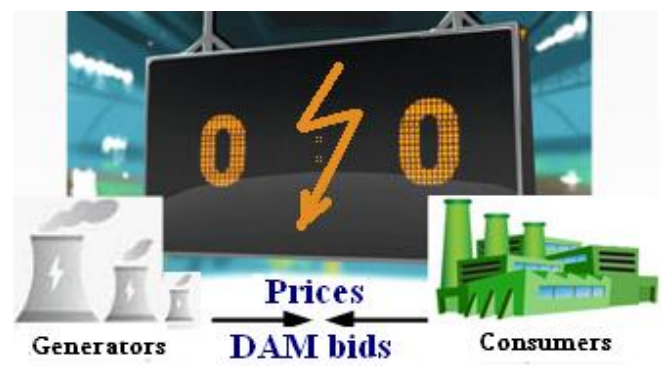

Fig. 2. "A game" of wholesale electric energy market participants.

For example, the buyer indicating 243 -stage pairs of "volume-prices in the DAM bid establishes the price value that he agrees to pay for the given volume of electric energy at a certain hour of day, trying to predict the prices values set by the generators in similar bids. If we consider the influence of participants' moves on each other in a day following the predicted one then these participants use the statistic values of DAM and BM prices of the previous day that in their turn have been determined by earlier moves made by all the EM participants (Fig. 3). 


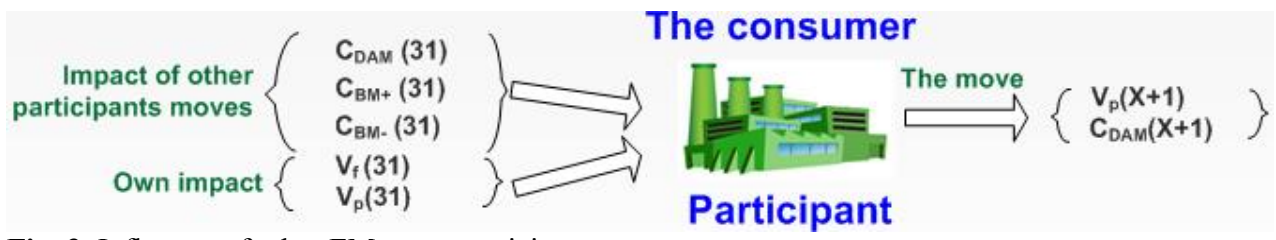

Fig. 3. Influence of other EM game participants.

Thus, the EM game is consistent, because the hourly price values received from the market Operators in accordance with Regulations $[10,11]$ indirectly depend on the previous moves made by all the participants. Taking into account the presence of uncertainty relative to other participants' bids placed on this current day it is possible to say the consistent game of the EM participants is a game with incomplete information in accordance with generally accepted classification. Presuming that the determination of each main analyzed parameters - type of price and electric energy volume ratios- is a nondeterministic experiment in which only one of the two mutually exclusive outcomes can be implemented (Fig. 4) for formal description of the outcome implementation process during the analyzed interval being equal to the EM settling period, it is possible to apply the tools of the theory of probability [12].

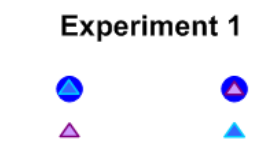

Outcome1 Outcome2

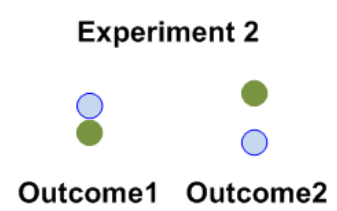

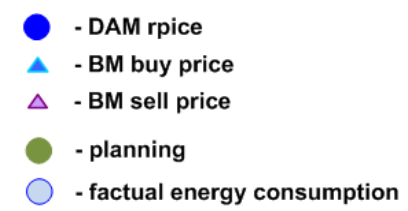

Fig. 4. Non deterministic experiments during determination of price and volume ratios.

Because the principle of optimality that is used to select a suitable alternative from a number of possible update values $V_{p}$ is unknown, it should be found by solving the selection task in accordance with the decision - making theory. The final decision will be a function of utility or profit. The target function of a model for solving the set task in opinion of a person making the decision can be described as follows [7]:

$$
\left\{\begin{array}{l}
\sum_{\mathrm{i}=1}^{\mathrm{N}} S_{D A M+B M}^{i}<\sum_{\mathrm{i}=1}^{\mathrm{N}} S{ }_{D A M+B M}, \\
\sum_{\mathrm{i}=1}^{\mathrm{N}} V_{i} \leq \sum_{\mathrm{i}=1}^{\mathrm{N}} V *_{i},
\end{array}\right.
$$

Where $S_{D A M+B M}^{i}$ is electric energy cost and $V_{i}$ is the amount of deviations after updating the planed electric energy consumption, $S{ }_{D A M+B M}^{*^{i}}$ is electric energy cost and $V{ }^{*}{ }_{i}$ is the amount of deviations before updating, $i$ is the number of hour, $\mathrm{N}$ is the number of hours within a reporting month.

Thus, in this case the decision-making task is a multi-criterion task: one criterion is to reduce electric energy cost and another one is to reduce the amount of deviations. However, considering the fact that the amounts of deviations can be expressed in their costs and presuming the EM-out-of-balance update fraction as the second type costs [11], the task becomes a single-criterion one: its criterion is electric energy cost.

Summarizing the abovementioned information makes it possible to say that in order to ensure that only the profitable BM transactions are made, the enterprise needs to solve the task of selecting such an update value for preliminarily calculated planned electric energy consumption task that will make the expected hourly profit prevail over the loss within this settling period by calculating the utility function based on one criterion. In order to calculate the utility function, it is necessary to consider all possible options in the electric 
energy price-to-volume ratios during updates of $V_{p}$. Let us repeat briefly the outcome of utility functions earlier calculated by the authors $[4,7]$ by an example of negative update function $F_{0}$, which makes planned volume $V_{p}$ go down to updated value $V_{c}$ based on 6 main price-to-volume ratios (Fig. 5). In this situation, the actual, uneven and uncontrolled consumption $V_{f}$ can assume any values. Let us remind that there are 12 possible options of price-to-volume ratios ( 6 for each update type).
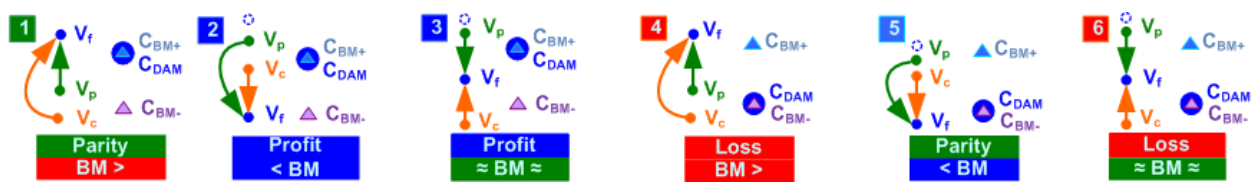

Fig. 5. Outcomes of experiments during determination of main price-to-volume ratios for negative update.

Let us record the possible profit for each option under consideration in accordance with probabilities of price-to-volume ratios obtained as a result of their analysis over 31 previous days - an interval being equal to the EM reporting period:

$$
\left\{\begin{array}{l}
P_{1} \mid P_{2}:-V \cdot C_{d i f} ; \\
P_{1} \mid P_{3}: V \cdot \Delta_{0}+V \cdot C_{d i f} ; \\
P_{1} \mid P_{4}:\left(V_{p}-V_{f}\right) \cdot \Delta_{0}+\left(V_{p}-2 \cdot V_{f}+V_{c}\right) \cdot C_{d i f} ; \\
1-P_{1} \mid P_{2}:-V \cdot \Delta_{1}-V \cdot C_{d i f} ; \\
1-P_{1} \mid P_{3}: V \cdot C_{d i f} ; \\
1-P_{1} \mid P_{4}:-\left(V_{f}-V_{c}\right) \cdot \Delta_{1}+\left(V_{p}-2 \cdot V_{f}+V_{c}\right) \cdot C_{d i f},
\end{array}\right.
$$

Where $P_{1}$ is probability of price ratio determination, when DAM and BM+ coincide; $P_{2}, P_{3}$ are probabilities that $V_{f}$ will be higher or lower than $V_{p}$ respectively; $P_{4}$ is probability that $V_{f}$ will be between $V_{p}$ and $V_{c} ; V$ is the update value equal to $\left|V_{p^{-}} V_{c}\right| ; \Delta_{0}, \Delta_{1}$ is an average difference in prices during the analyzed interval for BM price ratios of type 0 and 1 respectively; $C_{d i f}$ is an average price of deviations according to the enterprise unbalance share on the EM within the analyzed month [11].

The presence of $\mathrm{BM}$ price difference values $\Delta_{0}$ and $\Delta_{1}$ in these formulas is very important, because this BM price difference determines the profit or loss cost, which the economic effect for each day hour depends on:

$$
\text { Effect }_{-}^{+}=\left(C_{B M+}-C_{B M-}\right) * \mathrm{~V},
$$

The second possible parameter, as seen from the formula, is update size $V$.

Function $F_{0}$ is calculated using the full probability formula provided that less common options 3 and 6 are neglected (their total probability at average hourly consumption of enterprise equal to about $150 \mathrm{MWt} \cdot \mathrm{h}$ and update size equal to $5 \mathrm{MWt} \cdot \mathrm{h}$ amounts to $\approx 0.03$ ), transformation of $P_{3}$ into $\left(1-P_{2}\right)$ and reduction of $P_{4}$ :

$$
F_{0}=V \cdot\left(P_{1} \cdot d_{0}-P_{2} \cdot d_{1}+P_{1} \cdot P_{2} \cdot\left(d_{1}-d_{0}\right)-2 \cdot P_{2} \cdot C_{d i f}+C_{d i f}\right)
$$

The utility function of positive update $F_{l}$ is opposite by sign to function $F_{0}$. The update sign and volume of hourly electric energy consumption initially expressed by "even curve" is determined according to the values of utility functions $F_{0}$ and $F_{l}$ [7]: 


$$
\left\{\begin{array}{l}
F_{0}>0: V_{c}=V_{p}-V ; \\
F_{1}>0: V_{c}=V_{p}+V ; \\
F_{0}=F_{1}=0: V_{c}=V_{p} ;
\end{array}\right.
$$

An update carried out in accordance with the calculated values of these functions makes the profit prevail over loss within a reporting month in accordance with statistical stability property. In this situation it is worth noting the positive moment for the planned consumption update method. The BM prices BM $C_{B M^{+}}$and $C_{B M}$ are determined by the EM System Operator (SO) based on the calculation of price indicator $C_{\text {ind }}[11]$ :

$$
\begin{aligned}
& C_{B M+}=\max \left(C_{D A M} ; C_{\text {ind }}\right) \\
& C_{B M-}=\min \left(C_{D A M} ; C_{\text {ind }}\right)
\end{aligned}
$$

In case of negative update $V_{p}$, when it is expected that the price ratio of type 0 is established (with profitable buy on the BM), and the SO predictive model is stimulated towards a decrease in BM price-indicator and consequently towards an increase in probability of price ratios of expected type (Fig. 6).
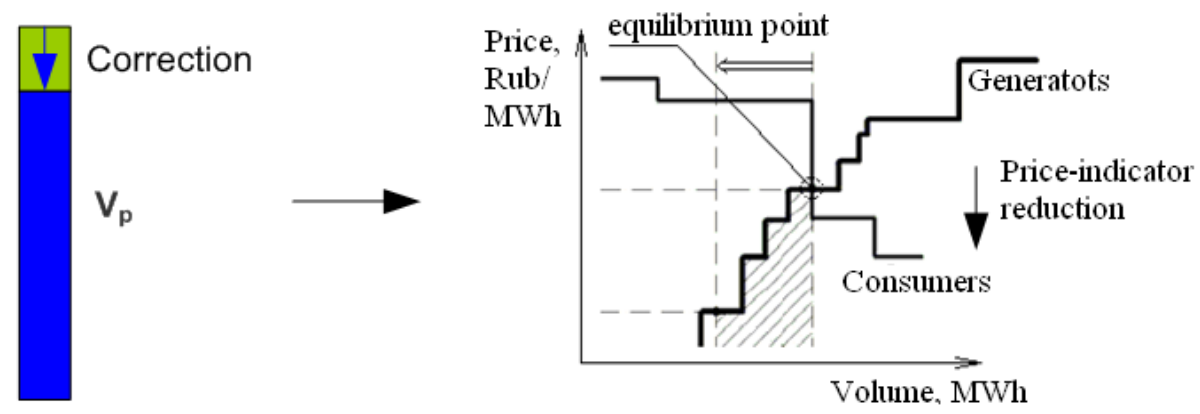

Fig. 6. Influence of negative update on profitable price ratios of type 0 .

And on the contrary, when $V_{p}$ is updated towards an increase in expectation of price ratios of type 1 (with profitable sale on $\mathrm{BM}$ ), the $\mathrm{SO}$ predictive model is stimulated towards an increase in BM price indicators and formation of price ratio of type 1 (Fig. 7). The influence of this factor depends on the enterprise's electric energy consumption share in its region and update size of its planned hourly electric energy consumption.
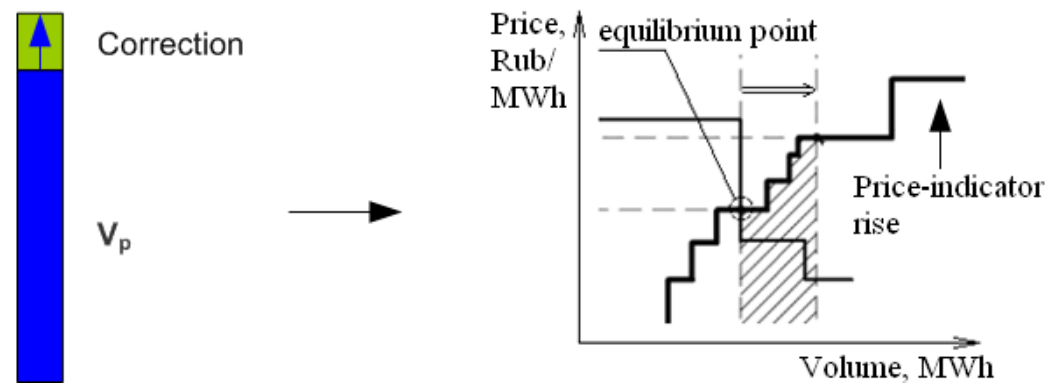

Fig. 7. Influence of positive update on profitable price ratio of type 1.

The DAM and BM price ratios are virtually the signals given by the SO to the consumer about how the generators are loaded at a certain hour and how electric energy should be consumed. That is why following these signals makes it possible to avoid unprofitable transactions made on the BM with no excessive load of inefficient generators and as a 
consequence, save non-renewable resources such as coal, gas, black oil, which helps solve environmental problems within an energy consumption region and the country as a whole.

\section{Algorithm of proposed method}

The scaled-up algorithm of this method, which is used to implement the proposed method is shown in Fig. 8. The actions described are carried out for each hour of day, and calculations can be made in parallel in this situation. It is presumed that the enterprise has runs a database on electric energy consumption $V_{p}, V_{f}$ and prices $C_{D A M}, C_{B M+}, C_{B M-}$ for each hour of day as deep as 31 days for the future period.

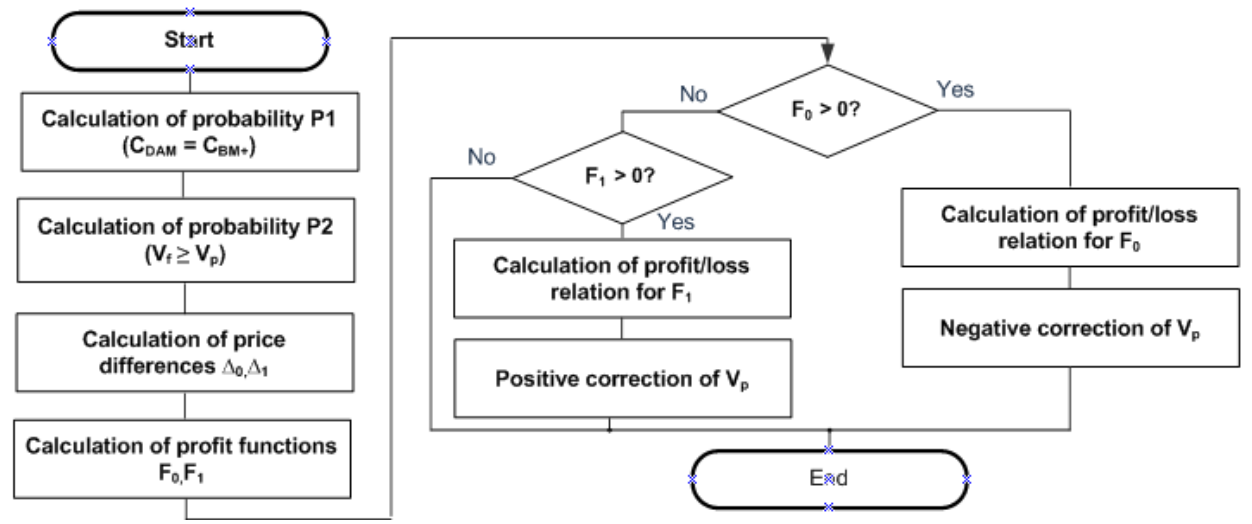

Fig. 8. Algorithm of digital method for updating of planned electric energy consumption.

The computer software operating in accordance with the described algorithm received state registration certificate in 2017 as per the established procedure.

\section{Method validation}

The software module in $\mathrm{C}++$ language implementing the above-mentioned algorithm operates within the electric energy data measuring complex of metallurgical enterprise operating in the Volgograd region. Every day the module analyzes the values of electric energy prices and volumes over the previous 31 days for every 24 hours, issuing recommendations for updating its planned electric energy consumption. Profit is determined by comparing the financial results of original and updated DAM bids (fig. 9).

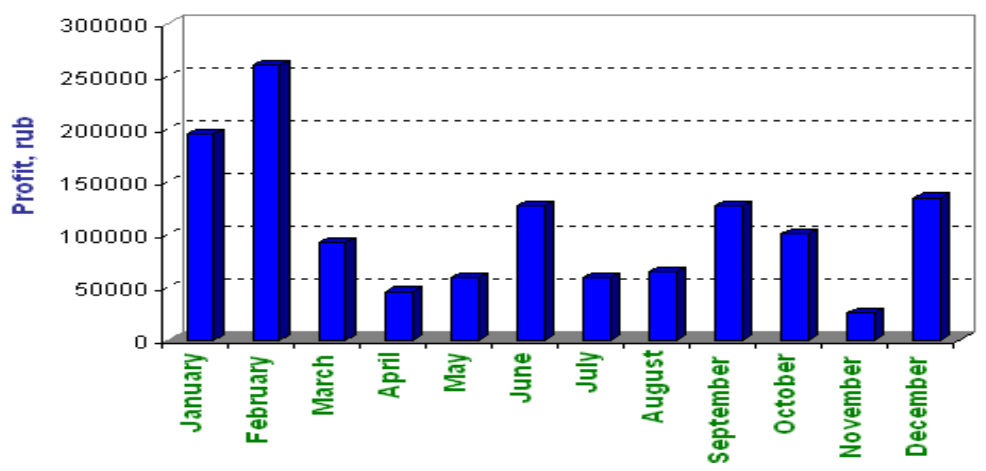

Fig. 9. Profit from recommendations of digital method broken down into months of 2017. 
Total profit gained from recommendations for updating the enterprise's planned electric energy consumption amounts to about $1.3 \mathrm{mln}$ rubles for 2017 .

Actual distribution of price-to-volume ratio experiment outcomes (Fig. 5) is shown in Fig. 10. As seen in the figure for the current software version the maximum quantity of outcomes corresponds to the option when the algorithm finds it difficult to choose an alternative for update, as a result the planned electric energy consumption remains unchanged.

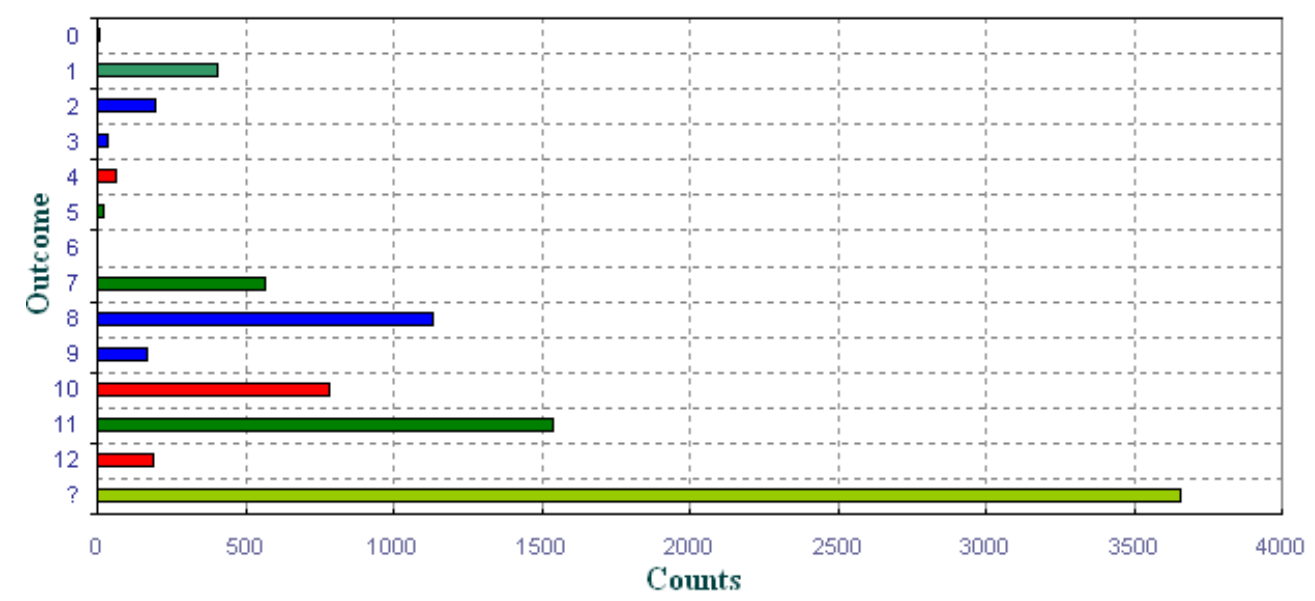

Fig. 10. Distribution of DAM and BM price options from 1.01.17 to 31.12.17.

Thus, with recorded economic effect there is a significant margin for further improving the algorithm by adjusting its main parameters.

\section{Conclusion}

The proposed digital method for updating the planned electric energy consumption allows solving the main task to be faced during planning of uneven, unregulated electric energy consumption by an industrial enterprise - reducing the electric energy consumption cost with no possible direct influence on the deviations of actual electric energy consumption from planned one. In this case the method is a step in creating a new high-tech industry using digital algorithms and means for optimizing the production process with minimum costs of all resources. In particular, the proposed digital algorithms allows enterprises that use it to be ahead on the developing prospective "Energynet" market, because these enterprises stop being passive energy consumers and become smart participants in the energy market, which makes it possible, among other issues, to minimize the cost of inhouse hourly deviations of planned electric energy consumption from actual one. In modern conditions of financial crisis it allows large industrial enterprises, many of which are cityforming ones and have to fill city and regional budgets, to have an additional high-tech tool to reduce their running costs and enhance their competitiveness. It is also important that updating the planned electric energy consumption in accordance with electric energy market signals results in no excessive load of inefficient generator and as a consequence saves non-renewable natural resources.

\section{References}

1. H.K. Alfares, M. Nazeeruddin, Int. J. of Sys. Sc., 33.1 (2002) 
2. P.V. Val, Short-term forecasting of power consumption of a mining enterprise in the conditions of the wholesale electricity and capacity market (SibFY, Krasnoyarsk, 2012)

3. B.I. Makoklyuev, V.F.Ech, Electric Power Plants, 5 (2005)

4. O.V. Russkov, S.E. Saradzhishvili, ICIEAM, 1-4 (2017)

5. S.S. Novikov, Technique of operational planning and management of power consumption of large-capacity electric steelmaking furnaces when operating in the wholesale electricity market (MEI, 2008)

6. V.Sh. Trofimova, A.V. Lipatnikov, Newspaper of G. I. Nosov MSTU, 3 (2014)

7. O.V. Russkov, S.E. Saradgishvili, IOP Conf. Ser.: Earth and Env. Sc., 90 (2017)

8. M. Harris, Inside the crystal ball: how to make and use forecasts (Wiley, New York, 2015)

9. A.P. Dzuba, Energy management of industrial enterprises (SUSU, Chelyabinsk, 2014)

10. DAM Regulati [online], Available at: https://www.npsr.ru/ru/regulation/joining/reglaments/1971 (2018)

11. BM Regulations [online], Available at: https://www.npsr.ru/ru/regulation/joining/reglaments/1975 (2018)

12. I. G. Chernorytsky, Methods of decision making (BXV-Petersburg, Saint Petersburg, 2005)

13. M. Shahidehpour, H. Yamin, Z. Li, Market operations in electric power systems: forecasting, scheduling and risk management (Wiley, New York, 2002) 